\title{
Computational Investigation of the Role of Active Site Heterogeneity for a Supported Organovanadium(III) Hydrogenation Catalyst
}

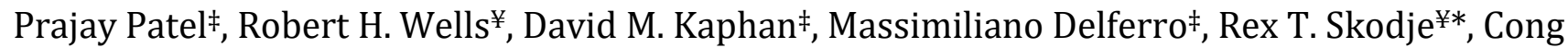 \\ Liü*
}

₹Chemical Sciences and Engineering Division, Argonne National Laboratory, Lemont, IL

¥Department of Chemistry, University of Colorado Boulder, Boulder, CO

DFT; kinetic Monte Carlo, Supported Organometallic Catalysts; Heterogeneity; Vanadium

\begin{abstract}
A crucial consideration for supported heterogeneous catalysts is the non-uniformity of the active sites, particularly for Supported Organometallic Catalysts (SOMCs). Standard spectroscopic techniques, such as X-ray absorption spectroscopy (XAS), reflect the nature of the most populated sites, which are often intrinsically structurally distinct from the most catalytically active sites. With computational models, often only a few representative structures are used to depict catalytic active sites on a surface, even though there are numerous observable factors of surface heterogeneity that contribute to the kinetically favorable active species. A previously reported study on the mechanism of a surface organovanadium(III) catalyst [(SiO)VIII(Mes)(THF)] for styrene hydrogenation yielded two possible mechanisms: heterolytic cleavage and redox cycling. These two mechanistic scenarios are challenging to differentiate experimentally based on the kinetic readouts of the catalyst are identical. To showcase the importance of modeling surface heterogeneity and its effect on catalytic activity, density functional theory (DFT) computational models of a series of potential active sites of [(SiO)VIII(Mes)(THF)] for the reaction pathways are applied in combination with kinetic Monte Carlo (kMC) simulations. Computed results were then compared to the previously reported experimental kinetic study: 1) DFT free energy reaction pathways indicated the likely active site and pathway for styrene hydrogenation; a heterolytic cleavage pathway requiring a bare tripodal vanadium site. 2) From the kMC simulations, a mixture of the different bond lengths from the support oxygen to the metal center was required to qualitatively describe the experimentally observed kinetic aspects of a supported organovanadium(III) catalyst for olefin hydrogenation. This work underscores the importance of modeling surface heterogeneity in computational catalysis.
\end{abstract}

\section{INTRODUCTION}

Supported catalysts synthesized via surface organometallic chemistry are promising systems in the production of petrochemicals and fine chemicals due to their unique properties in combining the advantages of homogeneous and heterogeneous catalysis, which are the selectivity and stability, respectively. ${ }^{1-4}$ When modeling supported catalysts, the non-uniformity, or heterogeneity, of catalytically active sites is one of the more challenging aspects to depict. To this extent, the heterogeneity of active sites has been understudied both experimentally and computationally since the overall observed activity is dominated by the catalytically most active sites while standard spectroscopic techniques target the most populated sites or the aggregate of all sites on the surface.

Surface heterogeneity has been shown to have an effect on catalysis. ${ }^{5}$ As an inherently dynamic process, simulating catalytic kinetics requires reliable mathematical models. Numerous kinetic studies are performed on defect-free surfaces through periodic density functional theory (DFT) and kinetic Monte Carlo (kMC) simulations. ${ }^{5-6}$ These models, which are simplistic for practical purposes tend to model catalytic sites as energetically equivalent with equal reaction barriers. However, the observed complexity of catalytic kinetics and surface heterogeneity breaks these models, and while studies have examined defected or alloyed surfaces $^{7-9}$ as well as stepped faces, ${ }^{10-12}$ a full scope of their effect on the kinetics is incomplete.

Amorphous silica (a-SiO 2 ), a common support for SOMC, exhibits significant heterogeneity in anchoring sites, which leads to an ensemble of local environments with different structures and activities upon grafting of organometallic precursors. ${ }^{13-14}$ Small variations, such as the bond distance and angle between a rigid silanol group and the metal atom, can lead to a normal distribution of reaction barriers during catalysis. Studies by Peters, Scott et al. illustrated a simplified model to calculate site-averaged grafting barriers of single atom catalysts on an $\mathrm{a}_{-} \mathrm{SiO}_{2}$ surface that yielded a distribution of the kinetically favorable active sites based on minor variations of the local environments of the grafting sites. ${ }^{15-19}$ Recently, Copéret et al. corroborates studying metal-surface interactions and surface heterogeneity for alkene metathesis tungsten catalysts as they found multiple tungsten sites on the silica surface with several different local environments and for propane dehydrogenation gallium catalysts through investigating the effect of silica surface 
strain. ${ }^{20-21}$ These studies underscore the need for more developed computational models and well-defined environments for describing surface heterogeneity in SOMC.

An $\mathrm{a}^{-\mathrm{SiO}_{2}}$ supported organovanadium(III) complex $\left[\left(\mathrm{SiO}_{2}\right) \mathrm{V}^{\mathrm{III}}(\mathrm{Mes})(\mathrm{THF})\right]$ was previously reported to efficiently catalyze the hydrogenation of olefins. ${ }^{22}$ Structureproperty correlations revealed that low valent states and site isolation are required for hydrogenation. A follow-up kinetic and isotope labeling study ${ }^{23}$ suggested that the catalytic reaction follows the heterolytic $\mathrm{H}_{2}$ cleavage mechanism, where $\mathrm{H}_{2}$ is activated on the $\mathrm{V}-\mathrm{O}$ bond to form a $\mathrm{V}(\mathrm{H})$ $(\mathrm{OH})$ species, while the $\sigma$-bond metathesis mechanism is excluded. ${ }^{22}$ While experimental characterization and kinetic study provided structural insights and critical kinetic behaviors of [( $\left.\left.\mathrm{SiO}_{2}\right) \mathrm{V}^{\mathrm{III}}(\mathrm{Mes})(\mathrm{THF})\right]$, it is particularly meaningful to gain molecular understanding of the formation of the catalytically relevant active sites and the reaction mechanisms as this will help uncover the role of active site heterogeneity in the reaction energetics and observed kinetics.

Therefore, using the experimental findings as reference, two dimensions of active sites heterogeneity are explored computationally in this work: 1) the surface heterogeneity based on bond elongation and coordination environment, and 2) considering the effects of various inhibitors to the catalytic activity, which allows the examination of a combination of potential active sites rather than a single active site. Utilizing a multidimensional approach to model surface and active site heterogeneity leads to a derivation of siteaveraged kinetics. The knowledge gained from this work could be adopted for future design of V(III) and other supported catalysts on silica and other amorphous inorganic support materials.

\section{COMPUTATIONAL METHODS}

\section{Electronic Structure}

a-SiO 2 has been extensively studied with DFT methods. ${ }^{6}$ 24-25 Two types of computational models have been commonly used for describing a-SiO 2 , periodic slab models, ${ }^{6,24-}$ 25 which use a unit cell representation of the surface, and cluster models $25-31$, a finite fragment of the solid. As amorphous surfaces have nonuniform composition and the need for more realistic and larger surface models increases, modeling amorphous supports with periodic slab models becomes increasingly challenging. On the other hand, computationally more efficient cluster and cage models have been utilized to study local active site environments of catalysts grafted onto amorphous silica. Previous computational studies $^{25-31}$ have shown that a cluster model of silica can represent its bulk structures well while keeping the surface strain on the cluster. Furthermore, silica clusters composed of different siloxane ring structures (silsequioxane) could be a good approximation of an a- $\mathrm{SiO}_{2}$ surface. ${ }^{26}$ Based on their success and previous use in our group to model SOMC, silsequioxane cages are utilized in this work due to the number of structures needed for the full mechanistic nature of this investigation.

All electronic structure calculations for the molecular structures and reaction energetics were performed using
Gaussian16.32 As shown in previous work, silsequioxane cages were shown to be adequate models for amorphous $\mathrm{SiO}_{2}$ in lieu of periodic DFT calculations. ${ }^{25}$ To model top monolayer relaxation in periodic models, the bottom half of the silica cluster $(6 \mathrm{H}, 110$, and $6 \mathrm{Si}$ atoms $)$ was kept frozen during the optimization and frequency calculation. All structures were optimized using the B3LYP33-34 density functional and CEP-31 $\mathrm{G}^{35-37}$ pseudopotential double- $\zeta$ basis set in the gas phase. For the free energy reaction profile, the Gibbs energy thermal corrections, single point energy, and frequency calculations are obtained using B3LYP with the $\mathrm{TZVP}^{38}$ basis set at $50^{\circ} \mathrm{C}$ to replicate experimental conditions. The previous experiments used dodecane as solvent, the effect of which was assumed to be small. Thus, solvents were not considered in these calculations.

\section{Kinetic Studies}

Kinetic Monte Carlo (kMC) applies Monte Carlo sampling towards catalytic chemistry through obtaining kinetic observables when the reaction environment plays a key role or when the reaction rates are non-uniform. ${ }^{39-41}$ Using kMC and the single-molecule description of kinetics, reaction profiles of each catalytic site can be explicitly viewed to interpret bulk properties of the whole system, which is a composition of the turnovers from the individual sites. This work examines the impact of multi-step reaction pathways that contain correlations among their distributed energetic barriers on the production pathway for styrene hydrogenation.

The rate of styrene hydrogenation is found using a simulation based on the free energies of the elementary reaction steps assuming that the amorphous support for the vanadium atoms form a static distribution of independent structures. The full reaction network for each structure thus corresponds to a specific set of reaction rate coefficients labeled by the structure index $q$. The turnover frequency (TOF) from a single structure can be calculated from a knowledge of the rate coefficients using conventional steady state kinetics. If the concentrations of styrene and hydrogen, $[\mathrm{S}]$ and $\left[\mathrm{H}_{2}\right]$, are regarded as constant parameters then the rate coefficients for the reaction step $i \rightarrow j$ is given by the standard Eyring form,

$$
k_{i, j}^{q}=\frac{k_{B} T}{h} e^{-\frac{\Delta G_{i, j}^{\ddagger, 0}(q)}{k_{B} T}} \cdot Z
$$

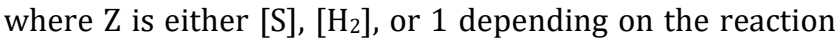
and $\Delta G_{i, j}^{\ddagger 0}(q)$ is the structure-dependent standard free energy barrier for $i \rightarrow \ddagger \rightarrow j$. The observed TOF is complicated in two ways: first the chemically distinct active sites can interconvert (see Scheme 4) yielding interlaced catalytic cycles that must be simultaneously considered, and second the active sites are statistically distributed over structures that yields different rate coefficients which must be folded together. In order to handle these issues, the TOF is formulated using a stochastic approach similar to that employed in modeling single molecular kinetics. ${ }^{42-44}$ The overall TOF is then a composite of contributions from specific chemical pathways that may exhibit different specific chemistry. ${ }^{45-46}$ The TOF for an arbitrary system is computed from the mean 
of the waiting time distribution as $v=\frac{1}{\langle t\rangle}$ where $t$ is the time interval between two product release events. The Markov chain of state-to-state transitions is explicitly generated using a standard kMC simulation. The averaging is done over all structures and active sites involving a large number of cycles. The reaction environment is simulated by creating approximately 1000 vanadium pre-catalysts in a solution of varying concentrations of styrene, $\mathrm{H}_{2}$, and THF molecules. The THF to catalyst ratio stays at a minimal $1: 1$ ratio due to the observed kinetics of THF as an inhibitor in previous work. Each catalytic site is treated as rigid so that the network energetic barriers are categorized by V-O bond distance. An equilibrium approximation was used for species that undergo rapid interconversion due to low barrier heights. The rejection-free Monte Carlo algorithm is used to stochastically simulate the kinetics. The reactants compete for adsorption onto the catalytic sites as detailed by the reaction scheme 4 . Upon product formation, the active sites are regenerated, and reactants are again allowed to adsorb when the catalyst site reaches the correct active state. An advantage of these kMC simulations is the tracking of individual sites to obtain detailed information such as waiting times between product formation and reaction-pathways chosen for reactants. Combined with methods from single- molecule studies this data can be invaluable when examining kinetic observables such as the total number of products formed during the experiment that will be explored in subsequent work. ${ }^{47}$

\section{RESULTS AND DISCUSSION}

1. Computational models of catalytic sites considering surface heterogeneity

Vanadation of partially dehydroxylated silica (at $200{ }^{\circ} \mathrm{C}$; $4.9 \mathrm{OH}$ per $\mathrm{nm}^{2}$ ) with the molecular organometallic $\left[\mathrm{V}(\mathrm{Mes})_{3}(\mathrm{THF})\right]^{22}$ yielded an air-sensitive, paramagnetic, dark purple material. Nuclear magnetic resonance (NMR)scale grafting experiment showed the protonolysis and release of two out of three mesityl ligands and negligible THF from the original molecular precursor. . X-ray Absorption Near-Edge Structure (XANES) spectra of the chemisorbed species were consistent with a $3+$ oxidation state for the $\mathrm{V}$ center. ${ }^{23}$ Based on these observations, three different plausible pre-catalyst structures formed from three distinct grafting sites on silica (Figure 1) are proposed of general formula ( $\equiv \mathrm{SiO})_{2} \mathrm{~V}(\mathrm{Mes})(\mathrm{THF})$ : a four-coordinate bipodal tetragonal site (1a), a tripodal trigonal bipyramid site with a silanol donor (1) $)$, and a tripodal trigonal bipyramid site with a siloxane donor (1c). In all three sites, the $V$ center is bound to a THF and a mesityl ligand.
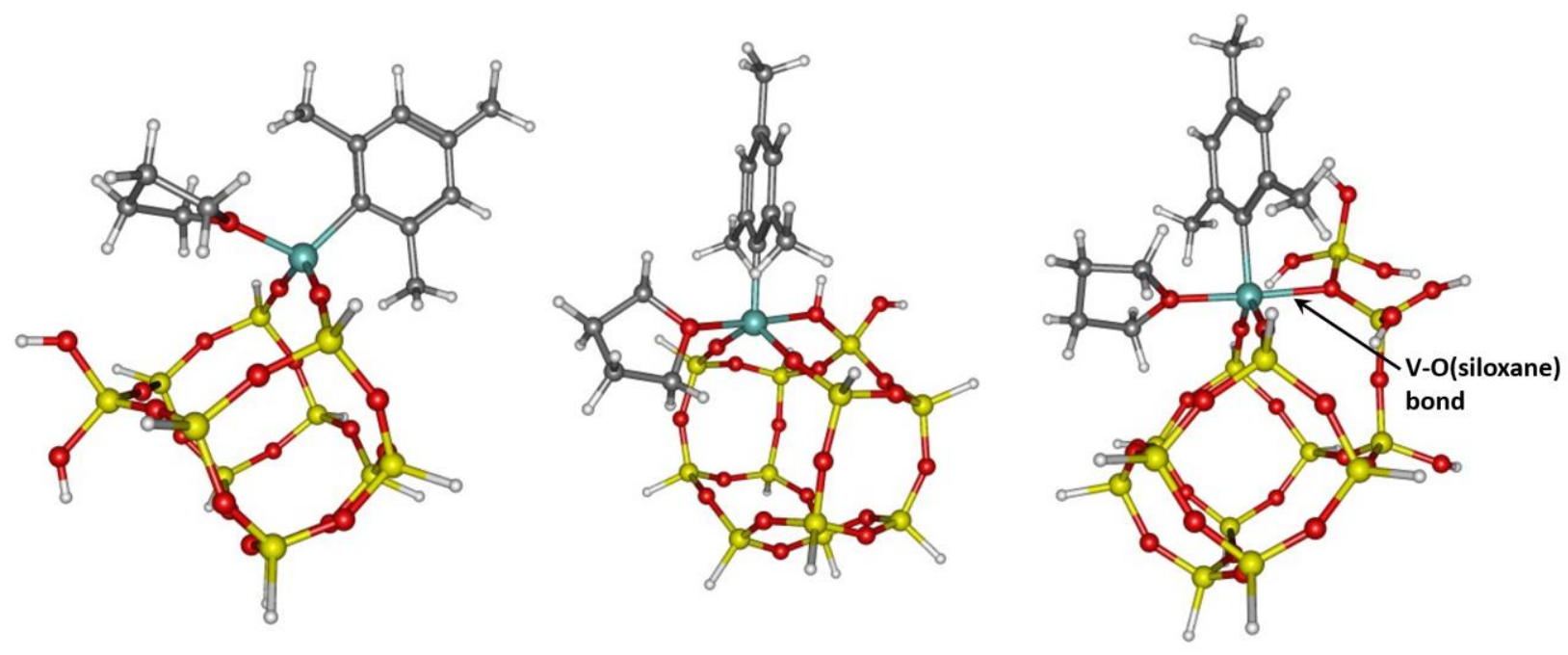

Figure 1. Cluster models of proposed pre-catalyst structures 1a (left), 1b (middle) and 1c (right) at the B3LYP/CEP-31G level of theory. $\mathrm{V}=$ Teal, $\mathrm{Si}=$ Yellow, $\mathrm{O}=$ Red, $\mathrm{C}=$ Gray, $\mathrm{H}=$ white.

For the cluster models of $\mathbf{1 a}$ and $\mathbf{1 b}$, the top half of the silica cluster can relax while keeping the bottom half $(6 \mathrm{H}$, 110 , and $6 \mathrm{Si}$ ) frozen during geometry optimization. However, for 1c, the two Si-O groups and the bottom half of the silica cluster are treated the same as for $\mathbf{1 a}$ and $\mathbf{1 b}$, while for the siloxane donor in the same plane as THF (V-O-Si), two scenarios are considered to represent the heterogeneity of the local geometries. The first scenario utilizes the rigidness of the neutral siloxane groups on the surface of amorphous silica, which infers the positions of the siloxane relative to the metal center; these environments are not likely to change during grafting and catalysis. The V-O(siloxane) distance is used as a parameter for a simplified description of the siloxane position relative to the metal center. Although other geometric parameters, such as bond angles and dihedrals, as well as the distance between two $\equiv \mathrm{SiO}-$ groups, could also play a role in describing the relative position of the siloxane, the $\mathrm{V}-\mathrm{O}$ (siloxane) distance is an effective simplification that is sufficient to demonstrate a qualitative trend in catalysis (vide infra). Based on the DFT optimized V-O (siloxane) distance for 1c $(2.29 \AA)$ in the fully re- 
laxed model, three rigid V-O (siloxane) distances are considered: 2.1, 2.3 and 2.5 $\AA$ as representative structures for compressed or elongated local environments in amorphous silica surface. In all calculations, the V-O distance is kept constant while the other geometric parameters are optimized. An alternative scenario considers the possibility that the donor siloxane could be on the edge or corner of the amorphous silica sample, which could provide more flexibility for the siloxane donor during grafting and catalysis given the extra degrees of freedom. In this case and for fundamental comparison with the first scenario, a system where the siloxane is unconstrained and therefore can fully relax during geometry optimization is also considered. As a result, there are six total proposed pre-catalyst systems, including $\mathbf{1 a}$, $\mathbf{1 b}$, and four structures for 1c corresponding to each individual V-O(siloxane) distance, i.e., fully relaxed (2.29 ̊), 2.1, 2.3 , and $2.5 \AA$ (Figure 1). In this work, the proposed active site structures are denoted by letters A-E to differentiate the active sites from the resting intermediates and transition states in this proposed mechanism.

\section{Formation of Catalytically Vanadium-Hydride Active Sites}

Previous experimental investigations showed that interaction of the pre-catalyst with $\mathrm{H}_{2}$ activates the supported vanadium center, forming a $\mathrm{V}-\mathrm{H}$ active site that catalyzes the hydrogenation of styrene and other unsaturated carbon-carbon bonds. Vanadium K-edge X-ray adsorption measurements following treatment of $\mathbf{1}$ with $\mathrm{H}_{2}$ are consistent with the formation of a new species with a reduced coordination number of non-hydrogen ligands, while maintaining the $3+$ oxidation state. ${ }^{23}$ Based on the XANES and
EXAFS characterization alone, it is uncertain whether the active sites formed from the pre-catalyst is a lower-coordinate $\mathrm{V}$ site or a V-H site because the hydride is not observable in EXAFS. Nevertheless, the experimental chemical reactivity ${ }^{22}$ suggests that a $\mathrm{V}-\mathrm{H}$ is generated on the surface. It is also unclear whether all the THF ligands remain on the metal center after $\mathrm{H}_{2}$ treatment. Therefore, all these possible structures are considered along with their associated reaction pathways for the six proposed pre-catalyst structures. 1a and $\mathbf{1 b}$ (Scheme 1) and 1c (Scheme 2) may undergo $\sigma$-bond metathesis with $\mathrm{H}_{2}$, which effects the cleavage of the mesityl ligand. Calculations (See SI for details) show that the four-coordinate $\mathrm{V}$ site (1a) is more energetically favorable in presence of THF ligand during the metathesis process, while the five-coordinate $V$ sites (1b and $\mathbf{1 c}$ ) requires the dissociation of THF before metathesis because of steric interactions. $\mathbf{1}$ and $\mathbf{1 b}$ form $\mathrm{V}-\mathrm{H}$ species $\mathbf{3}\left(\Delta \mathrm{G}^{\ddagger_{\text {intrinsic }}=}\right.$ $29.9 \mathrm{kcal} / \mathrm{mol})$ and $6\left(\Delta \mathrm{G}^{\ddagger_{\text {intrinsic }}}=28.6 \mathrm{kcal} / \mathrm{mol}\right)$, respectively, which are hypothesized to be the catalytically active sites (for the continued catalytic cycles, see Scheme 3), in agreement with experimental results. On the other hand, 1c first undergoes $\sigma$-bond metathesis to form a V-H (9a without THF or $\mathbf{9 b}$ with THF) that could react with the coordinated siloxane group on the support, resulting in the formation of a Si-H and a tripodal bare $\mathrm{V}$ site either with or without THF (11b without THF or 11a with THF, Scheme $2)$. Such a hydride transfer reaction has been reported previously for a silica supported $\mathrm{Zr}-\mathrm{H}$, in which $\mathrm{Si}-\mathrm{H}$ was observed via nuclear magnetic resonance (NMR) spectroscopy. 48

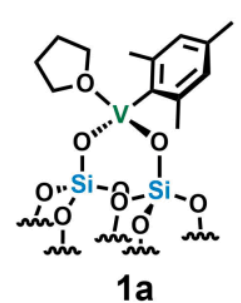

Pre-catalyst

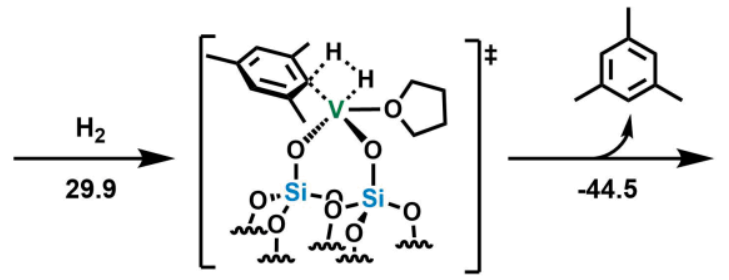

2

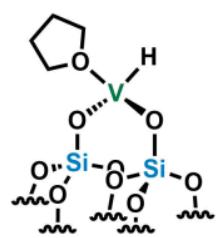

3

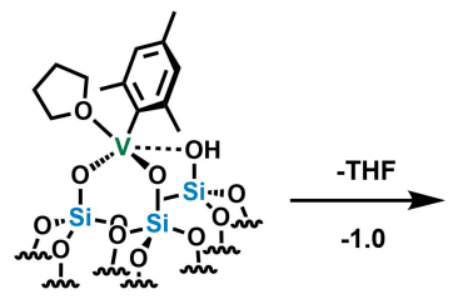

$1 \mathrm{~b}$

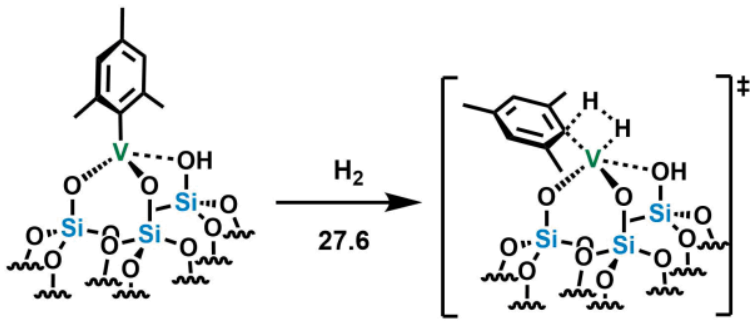

4
5
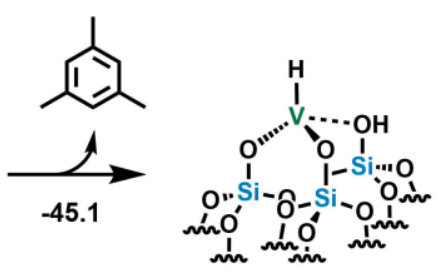

6

Pre-catalyst

Scheme 1. Proposed reaction pathways of the formation of potential active sites 3 (A) and 6 (B) for styrene hydrogenation. All free energies are reported in $\mathrm{kcal} / \mathrm{mol}$.

The four structures based on V-O(siloxane) distance for 1c described earlier are then considered to exploit the effect 
of surface induced strain on this pathway. As shown in Scheme 2, although the intrinsic barrier of the $\sigma$-bond metathesis (7 $\rightarrow$ 9a) decreases (from 27.1 to $22.0 \mathrm{kcal} / \mathrm{mol}$ ) with the increase of the $\mathrm{V}-\mathrm{O}$ (siloxane) distance, all the systems show a very similar apparent barrier (18.6-19.1 $\mathrm{kcal} / \mathrm{mol})$. The hydride transfer reaction $(\mathbf{9 b} \rightarrow \mathbf{1 1 b}$ or $9 \mathrm{a}$ $\rightarrow \mathbf{1 1 a})$ proceeds with very low barriers $(\leq 6.7 \mathrm{kcal} / \mathrm{mol})$ in all cases. This indicates the hydrides (either $\mathbf{9 b}$ or $9 \mathbf{9}$ ) resulting from the $\sigma$-bond metathesis are likely to have a very short lifetime and quickly transform to either a bare $V$ tripodal site (11a, C) or a THF bound V site $(\mathbf{1 1 b}, \mathbf{D})$ only if there is a proximally accessible siloxane for transfer. Note that the apparent barrier of $\mathbf{1 c}$ activation reaction is much lower than those of pre-catalysts $\mathbf{1 a}$ and $\mathbf{1 b}$ (29.9 and 27.6 $\mathrm{kcal} / \mathrm{mol}$, respectively), and the highest intrinsic barrier $(27.1 \mathrm{kcal} / \mathrm{mol}$ at $\mathrm{V}-\mathrm{O}($ siloxane $)=2.1 \AA)$ among the considered 1c structures is also lower than those of $\mathbf{1 a}$ and $\mathbf{1 b}$. This indicates that the activation of $\mathbf{1} \mathbf{c}$ by $\mathrm{H}_{2}$ could be kinetically more favorable than $\mathbf{1 a}$ and $\mathbf{1 b}$ due to destabilization by the binding of a fifth ligand.

Also notable from experimental observations, THF was free to bind or dissociate from the $V$ site at various reaction steps based on the reaction energetics. $\sigma$-bond metathesis $(\mathbf{7} \rightarrow \mathbf{9 a})$ occurs after the dissociation of THF $(\mathbf{1 c} \rightarrow \mathbf{7})$ for all V-O(siloxane) distances. However, for the hydride transfer reaction, sites with shorter $\mathrm{V}-\mathrm{O}$ (siloxane) distances (2.1 and relaxed) energetically favor the pathway without THF (9a $\rightarrow$ 10a $\rightarrow$ 11a), while those with longer V-O(siloxane) distances (2.3 and $2.5 \AA$ ) undergoes the pathway with coordinated THF (9a $\rightarrow$ 9b $\rightarrow \mathbf{1 0 b} \rightarrow \mathbf{1 1 b})$. This demonstrates the dynamic behavior of the THF coordination during the formation of individual catalytic sites. Although the resulting THF coordinated $\mathrm{V}$ site $\mathbf{( 1 1 b )}$ is thermodynamically more stable than the bare V site (11a) in all cases, it is hypothesized that both sites could exist under the reported experimental conditions ${ }^{23}$ and potentially be the active sites, due to the low concentration of THF (dissociated from the initial molecular precursor) in the chemisorption experiment. In addition to $\mathbf{C}$ (11a) and $\mathbf{D}$ (11b), it is also hypothesized that styrene, which presents a much higher concentration ( 290 times higher) than THF in the experiment, could act as a ligand for the bare $V$ site in $\mathbf{C}(\mathbf{1 1 a})$, forming the potential complex $\mathbf{3 1}$ (Scheme 2). Both the reaction pathways and energetics of the active site formation is affected by the $\mathrm{V}-\mathrm{O}$ (siloxane) distance and anticipated that the $\mathrm{V}-\mathrm{O}$ (siloxane) distance(s) could also be critical to the catalytic hydrogenation of styrene. 

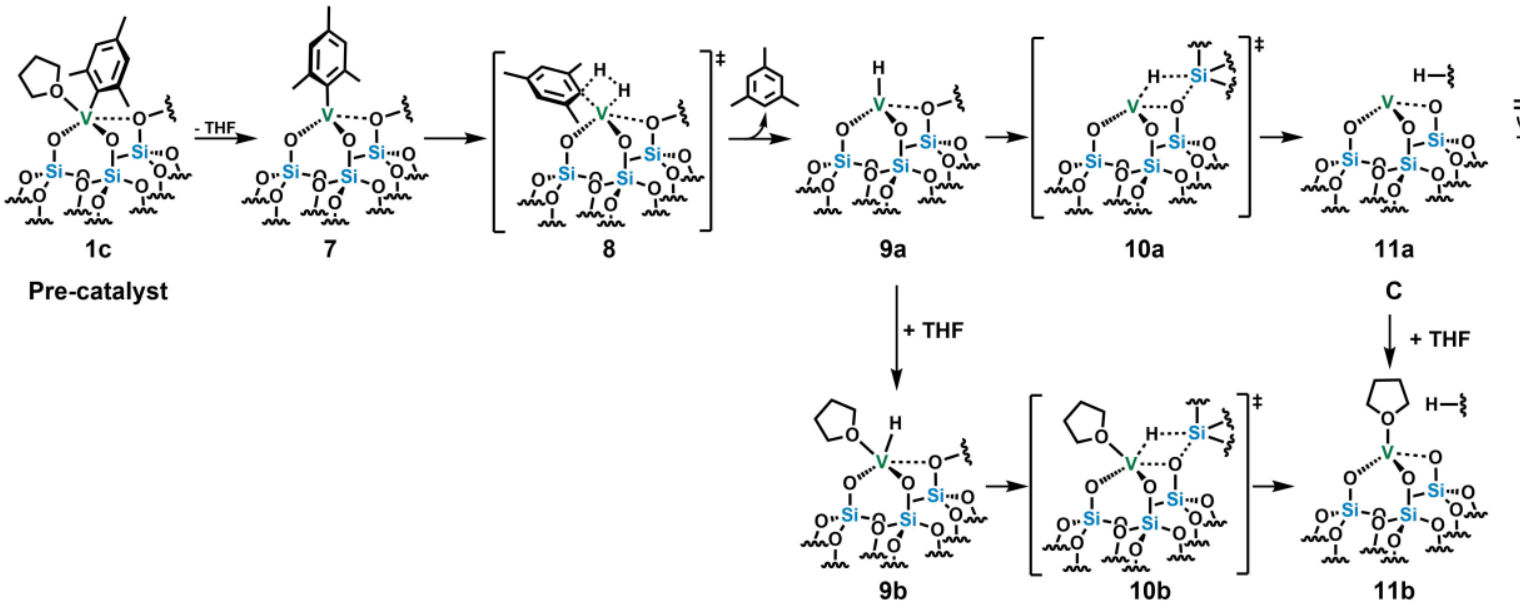

c

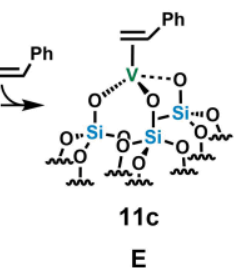

Pre-catalyst

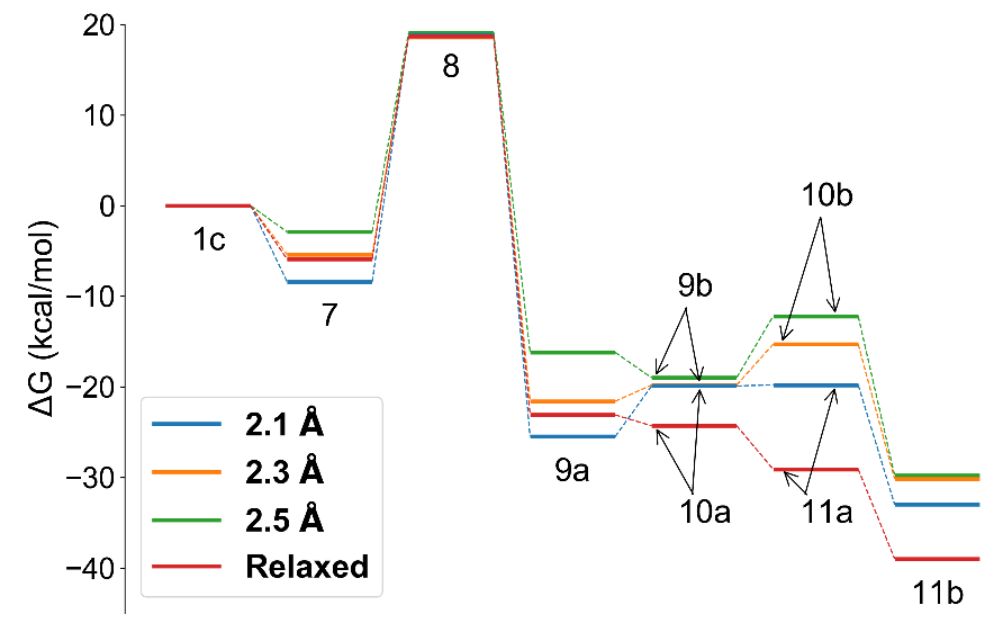

Scheme 2. (top) Proposed reaction pathways of the formation of active sites C (11a) and D (11b) for styrene hydrogenation. (bottom) The lowest free energy pathways for the formation of 11 b and 11 a as a function of the $\mathrm{V}-\mathrm{O}$ (siloxane) distance. The reaction free energies of higher energy pathways see Table $S 1$.

\section{Catalytic Hydrogenation Cycle}

Sigma Bond Metathesis (Active Sites A and B). Scheme 3 outlines the proposed catalytic cycles for styrene hydrogenation with $\mathbf{A}(\mathbf{3})$ and $\mathbf{B}(\mathbf{6})$, which are generated from activation of $\mathbf{1 a}$ and $\mathbf{1 b}$ by $\mathrm{H}_{2}$, respectively. These two systems are discussed together because they share a similar catalytic reaction mechanism. The reaction pathway of $\mathbf{3}$ undergoes migratory insertion $(\mathbf{3} \rightarrow \mathbf{1 3 a}$ ) into the benzylic carbon of styrene forming a V-alkyl 13a with a $22.5 \mathrm{kcal} / \mathrm{mol}$ barrier. An alternative pathway where the $\mathrm{V}$ center forms $\mathrm{a}$ bond with the homobenzylic carbon of styrene resulted in a slightly higher barrier (22.7 kcal/mol) (Figure S1). From intermediate 13a, hydrogenolysis of the alkyl $(\mathbf{1 3 a} \rightarrow \mathbf{3})$ regenerates 3 . In the case of 6 , the hydride insertion $(\mathbf{6} \rightarrow \mathbf{1 6 a})$ into the benzylic carbon of styrene results in 16 a via a 35.7 $\mathrm{kcal} / \mathrm{mol}$ barrier, and the hydrogenolysis of $16 \mathbf{a}(16 \mathbf{a} \rightarrow \mathbf{6})$ with a $34.5 \mathrm{kcal} / \mathrm{mol}$ barrier regenerates 6 (for alternative pathway see Figure S1). 
(a)
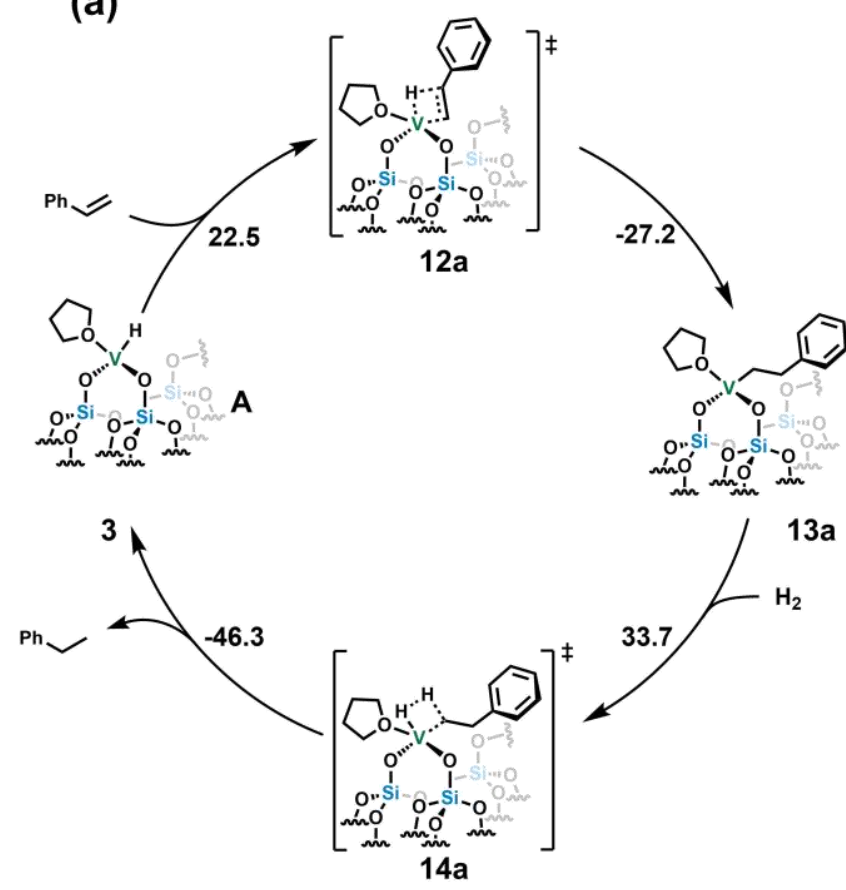

(b)
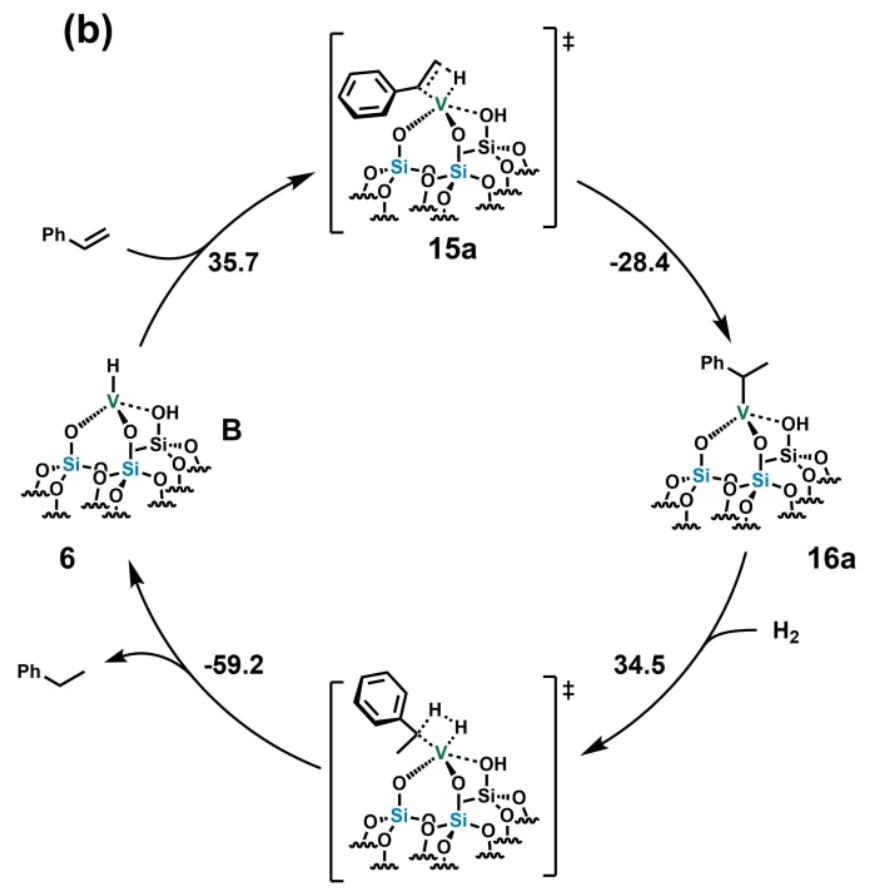

$17 \mathrm{a}$

Scheme 3. Proposed reaction pathways of the styrene hydrogenation with (a) 3 and (b) 6 as catalytic active sites. All free energies are reported in $\mathrm{kcal} / \mathrm{mol}$.

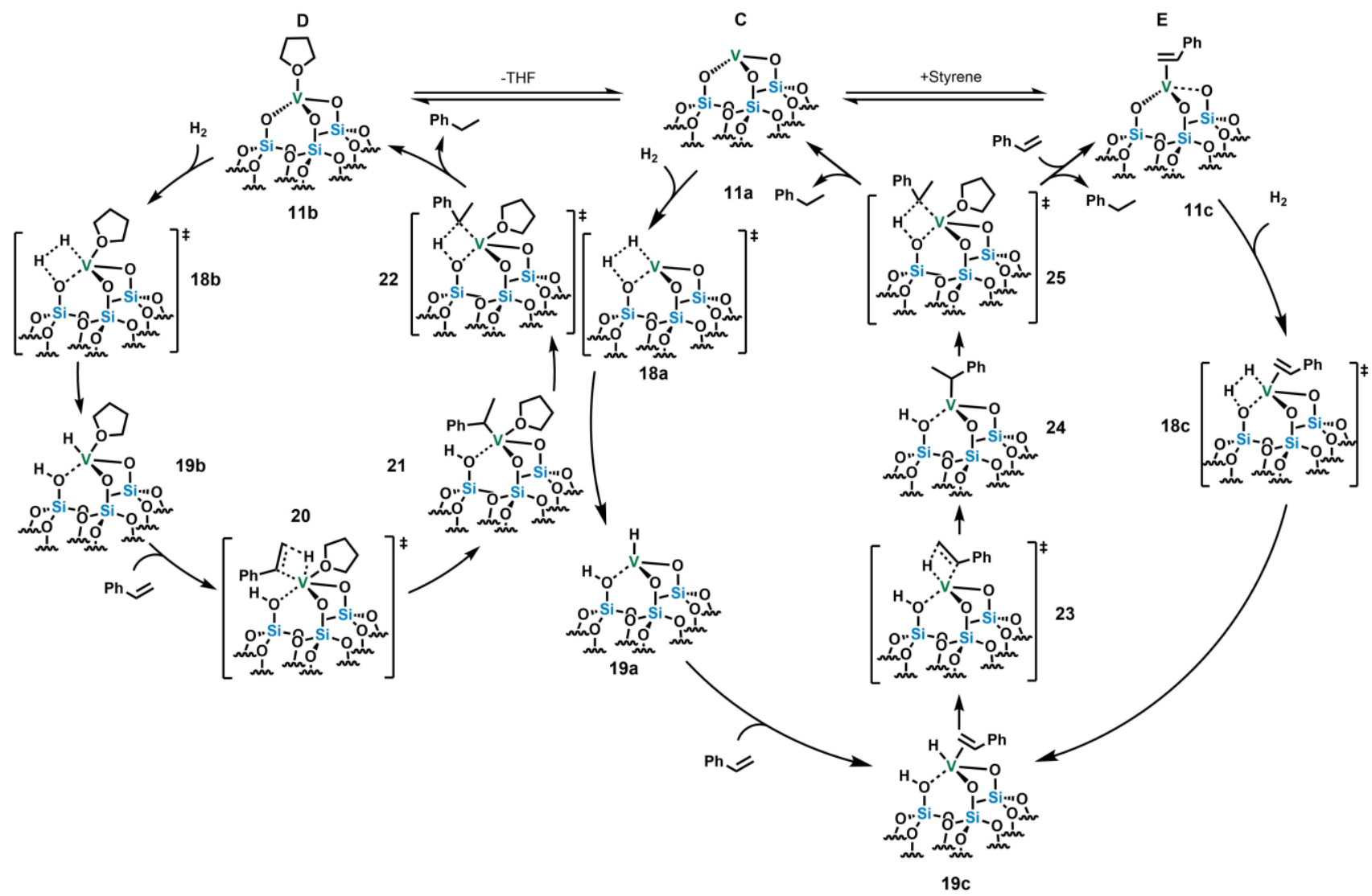

Scheme 4. Proposed styrene hydrogenation pathways for modeling active sites heterogeneity from 11a, 11b, and 11c (C, D and E). 
Heterolytic Bond Activation (Active Sites C, D, E). Scheme 4 shows the proposed reaction network for styrene hydrogenation catalyzed by 11a (C), 11b (D) and 11c (E). While individual reaction steps and intermediates vary depending on the active sites structure, all three active sites follow a similar catalytic mechanism: heterolytic cleavage of $\mathrm{H}_{2}$ followed by stepwise insertion into the styrene double bond and 1,2 elimination to regenerate the active site. Since $\mathbf{C}$ and $\mathbf{E}$ share the same pathway for the hydrogen transfer to styrene $(\mathbf{1 9 c} \rightarrow \mathbf{2 3})$, they are herein discussed together. As shown in Figure 2a and 2c, a general trend of the reaction free energies as a function of $\mathrm{V}-\mathrm{O}$ (siloxane) distance is observed: the longer the $\mathrm{V}-\mathrm{O}$ (siloxane) distance, the lower the pathway lies in the reaction energy diagram. The relaxed model shows the highest energy reaction pathway among all the models, presumably because its pre-catalyst activation undergoes the most exergonic reaction resulting in a relatively more stable (less active) active site than the three other model systems. For both $\mathbf{C}$ and $\mathbf{E}$, as the $\mathrm{V}-\mathrm{O}$ (siloxane) distance increases from 2.1 to $2.5 \AA$, the $\mathrm{H}_{2}$ cleavage barriers $(\mathbf{1 1 a} \rightarrow \mathbf{1 8 a}, 22.66,16.78,17.88,31.67 \mathrm{kcal} / \mathrm{mol}$, and 11c $\rightarrow$ 18c, 32.51, 27.04, 18.46, $40.77 \mathrm{kcal} / \mathrm{mol}$ for $2.1,2.3,2.5$, and relaxed respectively) decrease except for $\mathbf{1 1 a} \rightarrow \mathbf{1 8 a}$ at 2.3 and $2.5 \AA$ and forms more stable intermediates relative to the active sites. While the reaction barriers of the insertion of styrene into the V-H species $(\mathbf{1 9 c} \rightarrow \mathbf{2 3}, 8.63,8.92,7.64$, $8.70 \mathrm{kcal} / \mathrm{mol}$ for $2.1,2.3,2.5$, and relaxed, respectively) are not affected by the $\mathrm{V}-\mathrm{O}$ (siloxane) distance. Remarkably, the barriers of the 1,2-elimination (24 $\rightarrow \mathbf{2 5}, 20.51,23.87$, $26.25,19.39$ ) increase dramatically as the V-O(siloxane) distance increases, likely due to the stabilization of the Valkyl intermediate (24) and the elongation of the process of 1,2 elimination causing a contraction in the bond length. Eventually at V-O(siloxane) $=2.5 \AA$, the V-alkyl intermediate becomes the thermodynamically most stable state in the catalytic pathway, and comparable barriers are observed for 24 going forward (1,2-elimination, $24 \rightarrow$ 25, 26.25 $\mathrm{kcal} / \mathrm{mol}$ ) and going backward ( $\beta$-hydride elimination, 24 $\rightarrow 23,26.28 \mathrm{kcal} / \mathrm{mol}$ ). This indicates that at longer V-O(siloxane) distances corresponding to elongated coordination environments on the amorphous silica surface, the alkyl intermediate could act as the catalytic resting state, from which the reactions going forward and backward become reversible. The importance of this observation and its relevance to the experimental study will be discussed in the Section 3. The similarity between the pathways of $\mathbf{C}$ and $\mathbf{E}$ also presents in the changing trend of the rate-limiting step; as the $\mathrm{V}-\mathrm{O}$ (siloxane) distance increases, the rate limiting step switches from heterolytic $\mathrm{H}_{2}$ cleavage to 1,2-elimination. This switch occurs at $2.3 \AA$ for $\mathbf{C}$, and at $2.5 \AA$ A for $\mathbf{E}$. Note that the barrier heights of the heterolytic $\mathrm{H}_{2}$ cleavage is always lower for $\mathbf{C}$ than for $\mathbf{E}$ at each individual V-O(siloxane) distance. This indicates that styrene is acting as an inhibitor to the catalyst. Especially for catalytic systems with shorter V$\mathrm{O}$ (siloxane) distances, where the rate limiting step is the heterolytic $\mathrm{H}_{2}$ cleavage, $\mathbf{C}$ is likely to be kinetically more favorable than E. On the other hand, the catalytic pathway of D (Figure 2b) shows similar energetic trends to those of $\mathbf{C}$ and $\mathbf{E}$. However, the barrier heights and relative energies of the intermediates of $\mathbf{D}$ are all higher than those of the other two sites at each individual V-O(siloxane) distance. The highest barrier reaction step also changes when THF is bound to the tripodal vanadium active site, as in $\mathbf{D}$. The rate limiting step switches from the heterolytic $\mathrm{H}_{2}$ cleavage (11b $\rightarrow \mathbf{1 9 b})$ to the hydride insertion $(\mathbf{1 9 b} \rightarrow \mathbf{2 1})$ as the $\mathrm{V}-\mathrm{O}$ (siloxane) distance increases to $2.5 \AA$, at which the reversibility between the 1,2-elimination $(\mathbf{2 1} \rightarrow \mathbf{2 2})$ and $\beta$-hydride elimination $(\mathbf{2 1} \rightarrow \mathbf{2 0})$ from the V-alkyl $(\mathbf{2 1})$ is still not reached. These results suggest that THF also acts as an inhibitor to the catalyst, in agreement with the experiment, and the catalytic pathways of the THF bound $\mathbf{D}$ are not as energetically favorable as $\mathbf{C}$ and $\mathbf{E}$.

It is worth noting that the reaction barrier of heterolytic $\mathrm{H}_{2}$ cleavage decreases as the $\mathrm{V}-\mathrm{O}$ distance increases in general. This indicates that among the three $\mathrm{V}-\mathrm{O}$ bonds of a tripodal $\mathrm{V}$ site (C), $\mathrm{H}_{2}$ cleavage could likely occur on the longest $\mathrm{V}-\mathrm{O}$ bond. Here the V-O(siloxane) bond varies from 2.1 to $2.5 \AA$, longer than the other two V-O bonds (around $1.98 \AA$ ). Thus, in the considered models, it is reasonable to assume the $\mathrm{H}_{2}$ cleavage occurs on the $\mathrm{V}-\mathrm{O}$ (siloxane) bond. Additional calculations (Table S5) were carried out by increasing the $0-\mathrm{V}-0$ angle between the two 0 atoms on the surface excluding the siloxane donor, which increases the 0-0 bond distance. The results show that when the $\mathrm{V}$-O(siloxane) bond is set at $2.1 \AA$, the $\mathrm{H}_{2}$ cleavage barriers decreases as a function of the 0-0 distance except for $\mathbf{C}$, which fluctuated as the $\mathrm{O}-\mathrm{O}$ distance increased. This demonstrates another dimension of the site heterogeneity. Knowing the incompleteness of the current models, which did not consider the distribution of the other two V-O bond lengths as well as other variables among different local surface environments, a qualitative analysis will be the focus for the following kinetic modeling (Section 6).

For all the respective pathways shown in Figure 2, the fully relaxed model yielded the highest reaction barriers. These sites have a meta-stability caused by fully relaxing the system and are analogous to catalytic sites on the surface edges with more labile siloxane donors, which causes high barriers. Also, the ground state destabilization is a factor in the reaction barriers. This indicates that the hydrogenation is not favorable for catalytic sites on the edge of silica support. Thus, in the following sections the relaxed models were omitted from the discussions. 

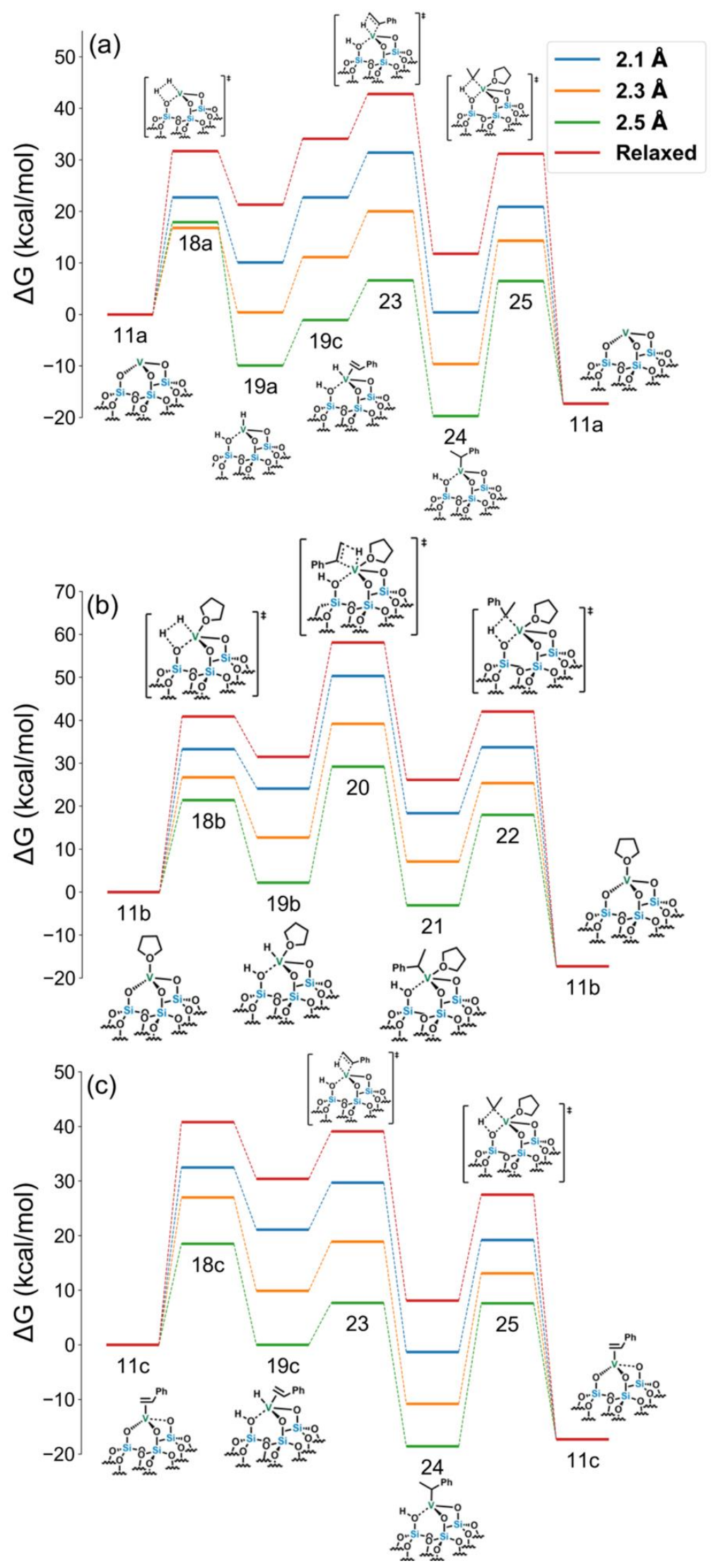

Figure 2. Free energy diagrams for the styrene hydrogenation with (a) C (b) D, and (c) E. The V-O(siloxane) distances are represented by lines (2.1 - blue, 2.3 - orange, 2.5 - green, and relaxed - red). All free energies are reported in $\mathrm{kcal} / \mathrm{mol}$. 


\section{Stability among Active Sites and Intermediate States}

The relative stability among $\mathbf{C}, \mathbf{D}$, and $\mathbf{E}$ is measured by the reaction free energies $(\Delta G)$ of the conversion among the three active sites (C, D, E); higher $\Delta \mathrm{G}$ yields a lower relative stability. The conversion of $\mathbf{C}$ to $\mathbf{D}$ and $\mathbf{E}$ results from the adsorption of THF and styrene, respectively. When the reaction free energy of adsorption/desorption of THF or styrene is small (a few $\mathrm{kcal} / \mathrm{mol}$ ), the conversion between two active sites becomes energetically accessible (reactants follow Curtin-Hammett principle $\left.{ }^{49-50}\right)$, and the conversion between the two could be facile. Such conversion reflects the dynamic change possibly occurring on a catalytically active sites during catalysis. As shown in Figure 3a, the relative stability of each active site decreases as the V-O(siloxane) distance increases, indicating the active sites at longer $\mathrm{V}$ $\mathrm{O}$ (siloxane) distances tend to be more reactive. This is consistent with the trends of the calculated reaction pathways, where lower barriers correspond to increasing the V-O(siloxane) distance. On the other hand, for each individual $\mathrm{V}$ $\mathrm{O}$ (siloxane) distance, $\mathbf{C}$ and $\mathbf{E}$ tend to have very similar stability while $\mathbf{D}$ (THF coordinated V site) is much more stable thermodynamically (by $13.2-16.5 \mathrm{kcal} / \mathrm{mol}$ ) than $\mathbf{C}$ and $\mathbf{E}$. The similar stability between $\mathbf{C}$ and $\mathbf{E}$ suggests that the binding of a styrene onto 11a (Figure 3a) is not particularly favorable. In other words, although styrene is present in high concentration under experimental conditions, it is not likely to become a strong inhibitor to the catalytic reaction. However, the much higher stability of THF coordinated D suggests that THF could become problematic to the catalytic reaction at high THF concentrations; the bare V sites (C) are likely to bind with THF prior to interacting with $\mathrm{H}_{2}$, leading to higher $\mathrm{H}_{2}$ cleavage barriers. Nevertheless, once the hydride intermediates (19a, 19b and 19c) are formed, the order of the stability changes (Figure $3 b$ ). The styrene coordinated hydride (19c) becomes less stable, and the difference in stability between the V-H (19a) and the THF coordinated hydride (19b) becomes minor $(\leq 3.8 \mathrm{kcal} / \mathrm{mol})$. This suggests that once the hydride is formed, THF could dynamically desorb from the $\mathrm{V}$ site, leading to much smaller barriers in the following catalytic pathway (Figure 2). At low THF concentrations, most active sites are likely to be the bare $\mathrm{V}$ sites (11a), which would have more impact on the overall kinetics in the catalytic reaction.
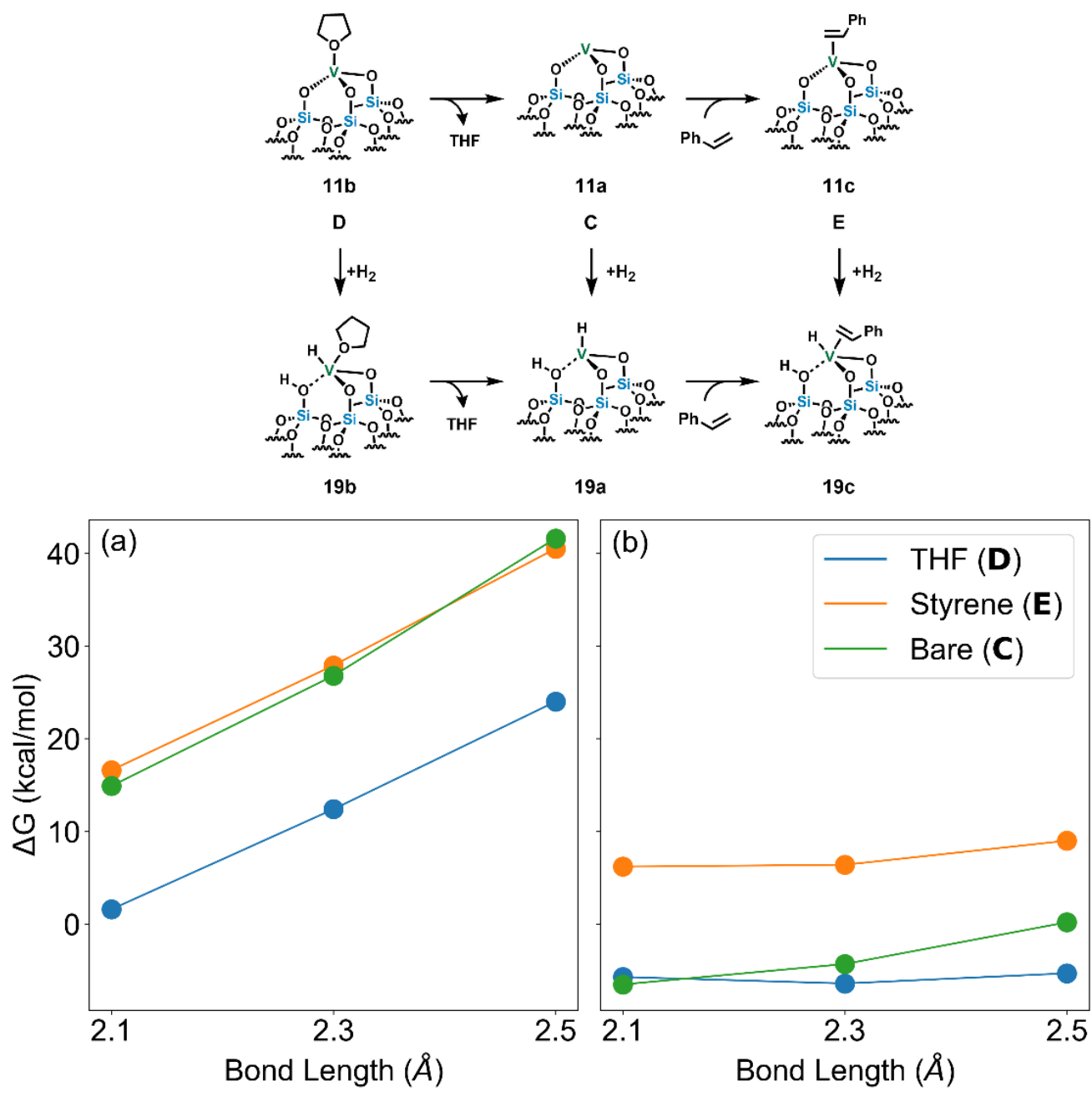

Figure 3. The relative stability of (a) active sites and (b) their hydrides (b). THF - blue, styrene - orange, bare V site - green. 
Table 1. Summary of computational relevance to the experimental mechanistic study. "Y" denotes that the computational results are consistent with experiments, while " $N$ " denotes an inconsistency.

\begin{tabular}{|c|c|c|c|c|c|c|}
\hline Pre-catalyst & & 1a & 1b & \multicolumn{3}{|c|}{ 1c } \\
\hline$\underset{23}{\text { Exp. characterization (NMR) }}{ }^{22-}$ & 4- or 5-coordinate with both THF and Mes ligands & Y & Y & \multicolumn{3}{|c|}{ Y } \\
\hline Active Site & & $\mathbf{A}$ & B & C & D & $\mathbf{E}$ \\
\hline $\begin{array}{l}\text { Exp. characterization }(\mathrm{XAS})^{22-} \\
23\end{array}$ & $\begin{array}{l}\text { Reduction of coordination number of non-hydrogen lig- } \\
\text { ands after } \mathrm{H}_{2} \text { treatment }\end{array}$ & $\mathrm{Y}$ & $\mathrm{Y}$ & Y & $\mathrm{Y}$ & Y \\
\hline \multirow{2}{*}{ Exp. Mechanistic Study ${ }^{23}$} & Deuterium incorporation pressure dependence & $\mathrm{N}$ & $\mathrm{N}$ & $\mathrm{Y}$ & $\mathrm{Y}$ & $\mathrm{Y}$ \\
\hline & Reversible hydride insertion & - & - & $2.5 \AA$ & - & $2.5 \AA$ \\
\hline
\end{tabular}

\section{Correlation with Experimental Mechanistic Study}

In the experimental study, ${ }^{22}$ the kinetic studies yielded saturation kinetics for styrene but near-first order under standard conditions, close to first order for $\mathrm{H}_{2}$, and first order in V. Using THF as an inhibitor was consistent with reversible dissociation at higher concentrations of THF, but deviation to more significant inhibition at lower levels of THF which could be consistent with poisoning the minority of THF deficient sites. NMR experiments during deuteration shows that the signal of protons on the $\alpha$-carbon of the styrene diminished more rapidly than the signals associated with the proton at the $\beta$-carbon or those associated with the aromatic ring, suggesting that at least some of deuterium was incorporated into the starting styrene by reversible hydride insertion. Furthermore, in an isotopic labeling experiment, deuterium pressure was found not to change the degree of deuterium incorporated between the $\alpha$ and $\beta$ carbon atoms in styrene.

The direct correlations between the previous experimental investigations and the present computational studies of the active sites and mechanisms are summarized in Table 1. In general, the structures of all the proposed precatalysts and active sites are consistent with the experimental characterization. However, the reaction mechanisms of $\mathbf{A}$ and $\mathbf{B}$ were found to have major inconsistency with the deuterium incorporation experiments. This suggests that the hydrogenolysis of the V-alkyl and the $\beta$-hydride elimination step would have the same order in hydrogen. ${ }^{23} \mathbf{A}$ and $\mathbf{B}$ both require an additional $\mathrm{H}_{2}$ in the hydrogenolysis step (Steps 13a $\rightarrow \mathbf{3}$ and $\mathbf{1 6 a} \rightarrow \mathbf{6}$, respectively, Scheme 3), which leads to a different order of reaction compared to the migratory instertion step (Steps $\mathbf{3} \rightarrow \mathbf{1 3 a}$ and $\mathbf{6}$ $\rightarrow$ 16a, respectively, Scheme 3). Using this in tandem with previous deuterium experiments indicating the dependence on the $\mathrm{D}_{2}$ pressure eliminates $\mathbf{A}$ and $\mathbf{B}$ from the list of potential active sites. The reaction mechanism of $\mathbf{C}, \mathbf{D}$, and E, on the other hand, has the same order for both steps, consistent with experiments. Recalling that some of the $\mathrm{D}_{2}$ was incorporated via reversible hydride insertion, this would require the reaction barrier of the hydrogen transfer $\mathbf{2 4} \rightarrow$ $\mathbf{2 5}$ for $\mathbf{C}$ and $\mathbf{E}, \mathbf{2 1} \rightarrow \mathbf{2 2}$ for $\mathbf{D})$ to the alkyl intermediate to be comparable or higher than that of the $\beta$-hydride elimination from the alkyl intermediate back to $\mathrm{V}-\mathrm{H}$ and styrene $(\mathbf{2 4} \rightarrow \mathbf{2 3}$ for $\mathbf{C}$ and $\mathbf{E}, \mathbf{2 1} \rightarrow \mathbf{2 0}$ for $\mathbf{D}$ ) as shown in Scheme 4 . In Figure 2, the calculations show a general trend between these two barriers as a function of the $\mathrm{V}-\mathrm{O}$ (siloxane) distance. As the V-O(siloxane) distance increases, the hydride transfer barrier increases while the $\beta$-hydride elimination barrier decreases. Furthermore, at V-O(siloxane) $=2.5 \AA, 24$ $\rightarrow \mathbf{2 3}(26.2 \mathrm{kcal} / \mathrm{mol})$ becomes comparable with $\mathbf{2 4} \rightarrow \mathbf{2 5}$ $(26.3 \mathrm{kcal} / \mathrm{mol})$ for $\mathbf{C}$ and $\mathbf{E}$, indicating the sites with longer $\mathrm{V}-\mathrm{O}$ (siloxane) distances could have a reversible hydride insertion. The computed barriers for the elongated $\mathrm{V}-\mathrm{O}$ (siloxane) bond (2.3 Å and $2.5 \AA$ ) are more consistent with experiment in that the first order dependence on styrene is consistent with the observed change in resting state, indicating that the elongated $\mathrm{V}-\mathrm{O}$ (siloxane) bond may be more favorable catalytically-there may be a distance between 2.3 and $2.5 \AA$ that satisfies both the observed kinetics and reversible hydride insertion. These computational results support the observed reversible hydride insertion, and further emphasize the importance to consider site heterogeneity.

These correlations with experiment eliminate $\mathbf{A}$ and $\mathbf{B}$ from the list of potential active sites. Among C-E, the calculated relative stability, as discussed in the previous subsection (Figure 3), shows that styrene binding onto either the $\mathbf{C}$ (to form $\mathbf{E}$ ) or the hydride intermediate does not increase the stability of the sites and leads to higher reaction barriers, indicating styrene is a poor inhibitor. Thus, in the following subsection, reaction pathways of $\mathbf{C}$ and $\mathbf{E}$, as well as the possible reversible conversion between these two active sites were considered for kinetic Monte Carlo simulations to illustrate the kinetic dependency of styrene, $\mathrm{H}_{2}$ pressure, and THF.

\section{Kinetic Monte Carlo Simulations of Rate Dependence}

Kinetic Monte Carlo (kMC) simulations were carried out to reproduce the experimental kinetic results and to validate the importance of the site heterogeneity. The kMC simulation results were focused on the experimental kinetic dependency of styrene, $\mathrm{H}_{2}$ pressure, and $\mathrm{THF}^{23}$ rather than quantitative agreement of the experimental rates. These simulations consider the effect of V-O(siloxane) distance, integrating both perspectives of surface heterogeneity and inhibiting species. 
(a)

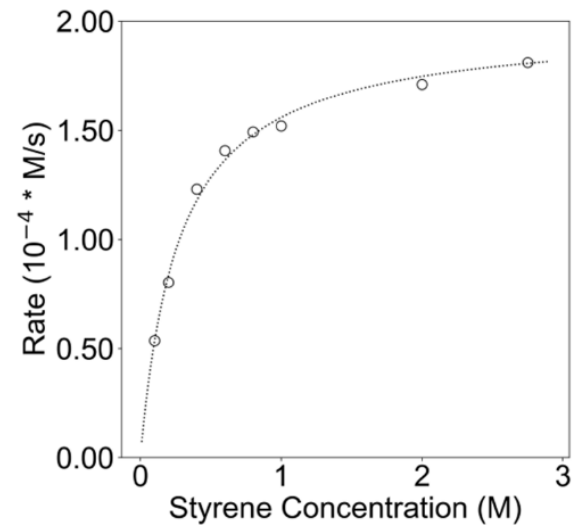

(d)

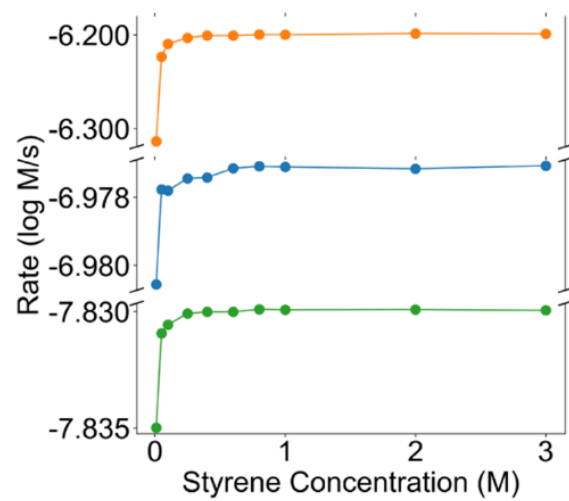

(g)

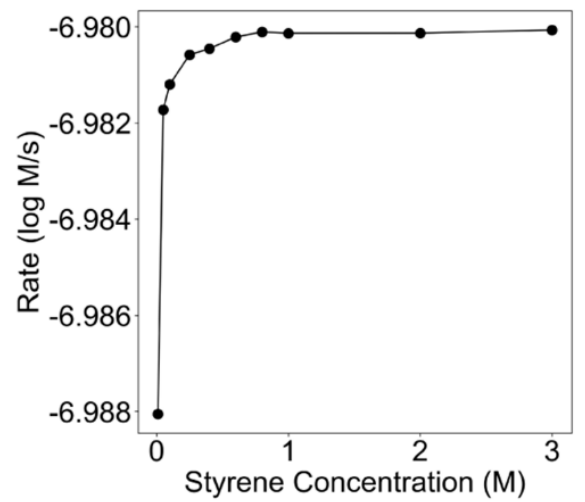

(b)

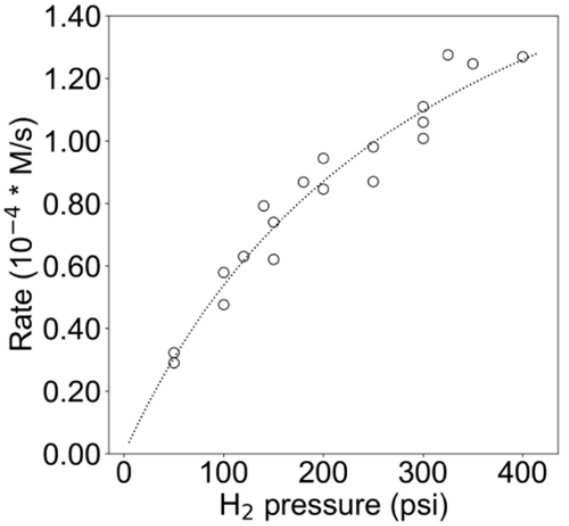

(e)

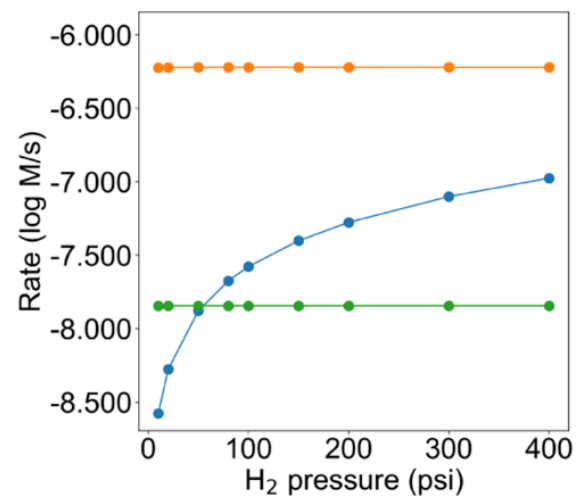

(h)

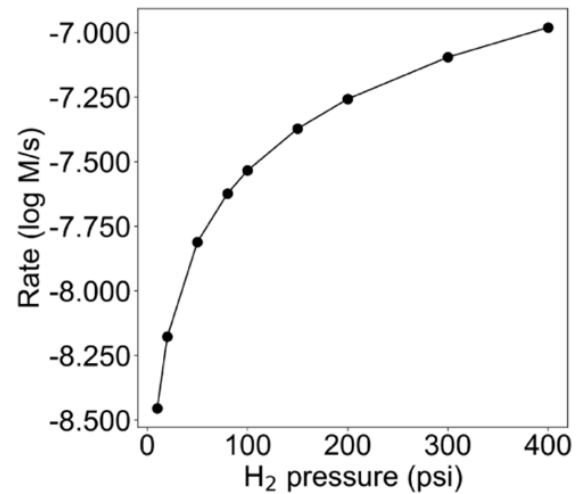

(c)

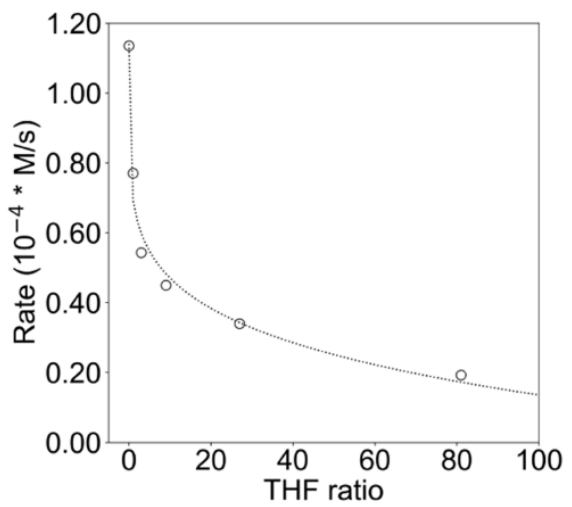

(f)

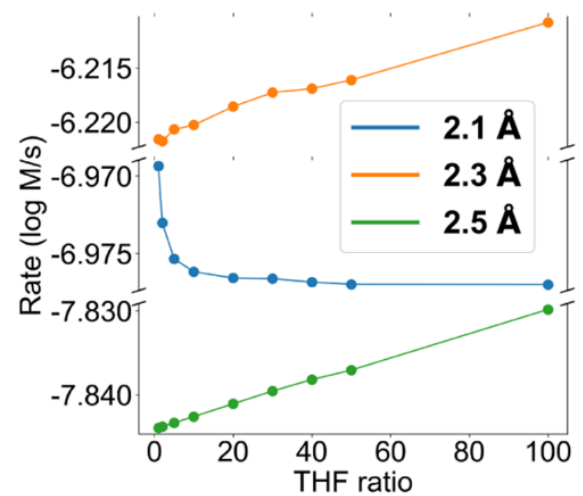

(i)

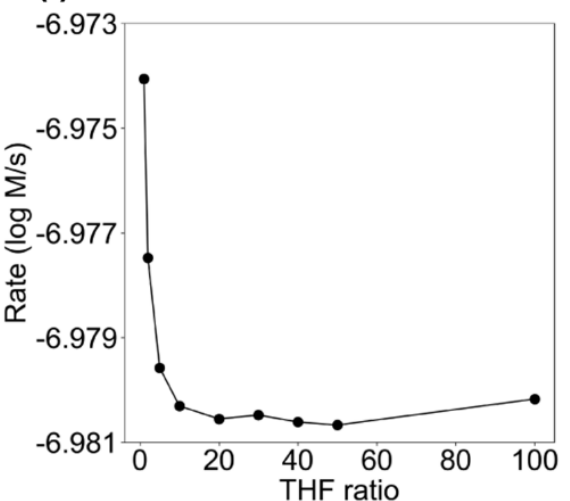

Figure 4. Experimental kinetic dependencies on (a) styrene, (b) $\mathrm{H}_{2}$ pressure, and (c) the ratio of THF for styrene hydrogenation from Ref. ${ }^{23}$. Kinetic Monte Carlo (kMC) calculated kinetic dependencies on (d) styrene, (e) $\mathrm{H}_{2}$ pressure, and (f) THF ratio at V- O(siloxane) $=2.1$ (blue), 2.3 (orange), and 2.5 (green) $\AA$. kMC calculated kinetic dependencies on (g) styrene, (h) $\mathrm{H}_{2}$ pressure, and (i) THF ratio using a linear combination of $92 \% 2.1,4 \% 2.3$, and $4 \% 2.5 \AA$ A. All kMC and experimental kinetic dependencies are reported in $\log \mathrm{M} / \mathrm{s}$ and $10^{-4} \mathrm{M} / \mathrm{s}$, respectively. The differences in magnitude between (a)-(c) (experimental) and (g)-(i) (kMC simulated) may be due to the underestimation of the true reaction barriers by $2-3 \mathrm{kcal} / \mathrm{mol}$ as well as the use of only three distances to model site heterogeneity. 
Figure 4a-c shows the experimental kinetic dependency of styrene, $\mathrm{H}_{2}$ pressure, and THF. ${ }^{23}$ The previous kinetic study $^{23}$ found a nonlinear dependence of the styrene concentration and hydrogen pressure on the production rates. The styrene dependence closely resembles a MichaelisMenten $^{51}$ like hyperbolic dependence. The hydrogen dependence, while not the pronounced hyperbolic dependence of styrene, is still non-linear. The transition rate between two states is given by the pseudo-first order rate coefficient:

$$
k(T)=\frac{k T}{h} \cdot \chi \cdot \exp \left(-\frac{\triangle G_{0}^{\ddagger}}{k T}\right)
$$

where $\triangle G_{0}^{\ddagger}$ is the free energy barrier and $\chi$ represents the concentration of a co-reagent, such as $\mathrm{H}_{2}$. An equilibrium approximation within the kMC was used for species that undergo rapid interconversion. The species in equilibrium are 11a and $11 b$ as well as their hydrides (19a and 19b). Recall that $\mathbf{1 1 b}$ is thermodynamically more stable than 11a, but the energy difference between the two hydrides is small ( $\leq$ $3.8 \mathrm{kcal} / \mathrm{mol}$ ) (Figure 3). The dependence of styrene concentration is first examined on the production rate across the three V-O(siloxane) distances (2.1, 2.3 and 2.5 $\AA$ ). kMC simulations of the relaxed model is not discussed here as it consistently showed the lowest rate of all scenarios (Details see Tables S6, S7 and S8). Figure 4d shows the simulated styrene dependence between $0.01 \mathrm{M}$ to $3.0 \mathrm{M}$. Reaction rates vary by orders of magnitude among the three $\mathrm{V}-\mathrm{O}$ (siloxane) distances. The $2.3 \AA$ model has the fastest production rate and shows the most qualitatively consistent concentration dependence with experiment (Figure 4a). It also is the data set which contains the highest amount of 11a (greater than $5 \%$ ). For the $\mathrm{H}_{2}$ dependence, there are noticeable similarities to styrene. The turnover rate was still the highest for the $2.3 \AA$ model with no apparent $\mathrm{H}_{2}$ dependence; however, $\mathrm{H}_{2}$ exhibited a greater dependence on the rate of product formation, which ranges over two orders of magnitude for the $2.1 \AA$ A model (Figure 4e). For this dependence to be exhibited in a simulation where the distributions of rate are present, the selectivity of the $2.3 \AA$ pathway must be larger than that of the $2.1 \AA$ pathway by a sizable amount. This indicates the number of the $2.3 \AA$ active sites are much bigger than that of the $2.1 \AA$ active sites. Both the styrene and $\mathrm{H}_{2}$ substrates will only have effects on the rate of turnover and that their concentrations are irrelevant to the catalytically relevant pathways. Examining the $2.1 \AA$ result for $\mathrm{H}_{2}$ production rates, the rate dependence does not plateau, which agrees qualitatively with the experimental $\mathrm{H}_{2}$ pressure dependence.

The relationship between THF concentration and production rate is the most complex of all three binding species. Experimentally, THF acts as an inhibitor; increasing the concentration of THF decreases the production rate. As illustrated earlier, THF as the inhibitor was proposed due to the high stability of $\mathbf{1 1 b}$ relative to $11 \mathrm{c}$ and $\mathbf{1 1 a}$ (Figure $3 \mathrm{a}$ ). However, the near-equilibrium between the THF coordinated hydride (19b) and the bare hydride (19a) (Figure 3b) indicates that THF could become bound and unbound multiple times during the reaction pathway. Such complexity was clearly reflected in the rate dependence of THF. The 2.3
$\AA$ model yielded the highest amount of production for the pathway of 11b. A slight inhibition effect is observed for the $2.1 \AA$ model (Figure 4f). However, the opposite correlation was observed for both the $2.3 \AA$ and $2.5 \AA$ models; increasing the THF concentration increased the reaction rate. Also, the THF concentration is directly correlated with a decrease in rate via $\mathbf{2 7} \mathbf{b}$ and an increase in production via 27 . In terms of the magnitudes of the rates, the kMC rates were two orders of magnitude lower than the experimental rates. This may be due to the underestimation of the true barrier heights by $2-3 \mathrm{kcal} / \mathrm{mol}$, the ratio of $\mathrm{H}_{2}$ to the catalyst, and the use of only a few $\mathrm{V}-\mathrm{O}$ (siloxane) distances as different $\mathrm{V}$ $\mathrm{O}$ (siloxane) distances changed the rate by an order of magnitude, further driving the point of modeling site heterogeneity. Overall, with both the $2.1 \AA$ and $2.3 \AA$ models in parts showing qualitative trends consistent with experimental kinetic profiles indicates that there is a dynamic distribution of contributions from different active site structures to the overall observed reaction rate for styrene hydrogenation.

When using a linear combination of the simulated kinetic profiles for each $\mathrm{V}-\mathrm{O}$ distance (Figure $4 \mathrm{~g}$-i), the reaction rate profiles qualitatively align with the experimentally observed kinetics. This was achieved with a linear combination of $92 \%$ of the $2.1 \AA$ model with $4 \%$ of the $2.3 \AA$ and 2.5 $\AA$ models each. Note that this ratio may not necessarily reflect the full scope that surface heterogeneity has on the observed kinetics, and this also conflicts with the DFT barriers, where the $2.5 \AA$ model is the most consistent with experimental kinetics. As shown in the DFT study, the positions of the other oxygen donors besides the siloxane on the support can also change the reaction barriers. Therefore, to better describe active site heterogeneity and reaction kinetics of styrene hydrogenation with the present computational models, inclusion of a variety of geometric parameters (e.g., all V-O bond lengths and angels) and their distributions are required. The kMC results using the current models are meaningful in the qualitative fashion, demonstrating an effective strategy and highlighting the importance to consider active sites heterogeneity in studying catalytic reaction mechanisms for SOMC.

\section{CONCLUSIONS}

Overall, this work showcases investigating active site heterogeneity through a multidimensional computational approach. The reaction pathways computed via DFT identified the more likely active sites, among which the calculated relative stability provides critical insights to the dynamic conversion among different sites during catalysis. For styrene hydrogenation, this pertains to the behavior of THF as an inhibitor and reactivity of the catalyst post- $\mathrm{H}_{2}$ treatment. The proposed mechanism for styrene hydrogenation follows the heterolytic cleavage mechanism with $\mathrm{H}_{2}$ acting as the inhibitor in the most energetically favorable pathway.

From the dimension of surface heterogeneity, the fully relaxed model is not necessarily a valid model to represent all the active sites based on both DFT and kinetic analysis. This was due to the constrained bond lengths, which mimics the rigidness of the silica support, that resulted in a ground state destabilization and lower reaction barriers whereas the ground state stabilization in turn yielded high reaction 
barriers for catalysis, whereas. The kinetic Monte Carlo simulations of varying reactant concentrations indicated that using a linear combination of the profiles for all three $\mathrm{V}$ $\mathrm{O}$ (siloxane) bond length $(2.1,2.3$, and $2.5 \AA)$ for the catalyst models were required to gain a qualitative understanding of the reaction kinetics, further emphasizing the importance to model surface heterogeneity.

From both the DFT and kMC perspectives, the qualitative accuracy using the present catalyst models with respect to the previous experimental work illustrates the importance of modeling surface heterogeneity when developing theoretical approaches to understanding single site heterogeneous catalysts. Yet, to gain quantitative accuracy for catalytic kinetics, more rigorous computational models derived from multiscale computational modeling need to be developed.

\section{ASSOCIATED CONTENT}

\section{SUPPORTING INFORMATION}

$\mathrm{XYZ}$ files for all the catalyst structures used for the reaction pathways. Additional schematics of reaction pathways. Tabular data for the kinetic Monte Carlo simulations.

\section{AUTHOR INFORMATION}

\section{Corresponding Author}

*Rex.Skodje@colorado.edu

*congliu@anl.gov

\section{Author Contributions}

Authors P.P and C.L are responsible for the DFT calculations. Authors R.W. and R. T. S. are responsible for the kinetic Monte Carlo calculations. Authors D.M.K and M.D. are responsible of the experimental data. All authors approve of the final manuscript.

\section{ACKNOWLEDGMENT}

This work was supported by the U.S. Department of Energy (DOE) Office of Basic Energy Sciences, Division of Chemical Sciences, Geosciences, and Biosciences, Catalysis Science Program under Contract DE-AC02-06CH11357 (Argonne National Laboratory). All DFT calculations were performed using the computational resources at provided by the Laboratory Computing Resource Center (LCRC) at Argonne National Laboratory (ANL). The work of R.H.W and R.T.S was supported by the National Science Foundation through grant CHE-1664555.

\section{ABBREVIATIONS}

DFT, Density Functional Theory; kMC, Kinetic Monte Carlo; SOMC, supported organometallic catalysts;

\section{REFERENCES}

1. Coperet, C.; Comas-Vives, A.; Conley, M. P.; Estes, D. P.; Fedorov, A.; Mougel, V.; Nagae, H.; Nunez-Zarur, F.; Zhizhko, P. A., Surface Organometallic and Coordination Chemistry toward Single-Site Heterogeneous Catalysts: Strategies, Methods, Structures, and Activities. Chem Rev 2016, 116 (2), 323-421.
2. Pelletier, J. D.; Basset, J. M., Catalysis by Design: Well-Defined Single-Site Heterogeneous Catalysts. Accounts of Chemical Research 2016, 49 (4), 664-77.

3. $\quad$ Samantaray, M. K.; Pump, E.; Bendjeriou-Sedjerari, A.; D'Elia, V.; Pelletier, J. D. A.; Guidotti, M.; Psaro, R.; Basset, J. M., Surface organometallic chemistry in heterogeneous catalysis. Chemical Society Reviews 2018, 47 (22), 8403-8437.

4. Witzke, R. J.; Chapovetsky, A.; Conley, M. P.; Kaphan, D. M.; Delferro, M., Nontraditional Catalyst Supports in Surface Organometallic Chemistry. ACS Catal 2020, 10 (20), 11822-11840.

5. $\quad$ Murzin, D. Y., On Surface Heterogeneity and Catalytic Kinetics. Industrial \& Engineering Chemistry Research 2005, 44 (6), 1688-1697.

6. Tielens, F.; Gierada, M.; Handzlik, J.; Calatayud, M., Characterization of amorphous silica based catalysts using DFT computational methods. Catalysis Today 2020, 354, 3-18.

7. Andersen, M.; Plaisance, C. P.; Reuter, K., Assessment of mean-field microkinetic models for CO methanation on stepped metal surfaces using accelerated kinetic Monte Carlo. J Chem Phys 2017, 147 (15), 152705.

8. Bray, J. M.; Schneider, W. F., First-Principles Analysis of Structure Sensitivity in NO Oxidation on Pt. ACS Catal 2015, 5 (2), 1087-1099.

9. Stamatakis, M.; Chen, Y.; Vlachos, D. G., First-PrinciplesBased Kinetic Monte Carlo Simulation of the Structure Sensitivity of the Water-Gas Shift Reaction on Platinum Surfaces. The Journal of Physical Chemistry C 2011, 115 (50), 24750-24762.

$10 . \quad$ Holby, E. F.; Wu, G.; Zelenay, P.; Taylor, C. D., Structure of $\mathrm{Fe}-\mathrm{Nx}-\mathrm{C}$ Defects in Oxygen Reduction Reaction Catalysts from First-Principles Modeling. The Journal of Physical Chemistry $C$ 2014, 118 (26), 14388-14393.

11. Lang, S. M.; Bernhardt, T. M., Gas phase metal cluster model systems for heterogeneous catalysis. Phys Chem Chem Phys 2012, 14 (26), 9255-69.

12. Li, H.; Evans, E. J.; Mullins, C. B.; Henkelman, G., Ethanol Decomposition on Pd-Au Alloy Catalysts. The Journal of Physical Chemistry C 2018, 122 (38), 22024-22032.

13. Thomas, J. M.; Raja, R.; Lewis, D. W., Single-Site Heterogeneous Catalysts. Angewandte Chemie International Edition 2005, 44 (40), 6456-6482.

14. Gajan, D.; Copéret, C., Silica-supported single-site catalysts: to be or not to be? A conjecture on silica surfaces. New Journal of Chemistry 2011, 35 (11).

15. Peters, B.; Scott, S. L., Single atom catalysts on amorphous supports: A quenched disorder perspective. Journal of Chemical Physics 2015, 142 (10), 104708.

16. Goldsmith, B. R.; Peters, B.; Johnson, J. K.; Gates, B. C.; Scott, S. L., Beyond Ordered Materials: Understanding Catalytic Sites on Amorphous Solids. ACS Catal 2017, 7 (11), 7543-7557.

17. Peters, B., Catalysis. In Reaction Rate Theory and Rare Events Simulations, Peters, B., Ed. Elsevier: Amsterdam, 2017; pp 79-128.

18. Khan, S. A.; Vandervelden, C. A.; Scott, S. L.; Peters, B., Grafting metal complexes onto amorphous supports: from elementary steps to catalyst site populations via kernel regression. React Chem Eng 2020, 5 (1), 66-76.

19. Vandervelden, C. A.; Khan, S. A.; Scott, S. L.; Peters, B., Site-averaged kinetics for catalysts on amorphous supports: an importance learning algorithm. React Chem Eng 2020, 5 (1), 77-86. 20. Pucino, M.; Liao, W. C.; Chan, K. W.; Lam, E.; Schowner, R.; Zhizhko, P. A.; Buchmeiser, M. R.; Copéret, C., Metal-Surface Interactions and Surface Heterogeneity in 'Well-Defined' Silica-Supported Alkene Metathesis Catalysts: Evidences and Consequences. Helvetica Chimica Acta 2020, 103 (6).

21. Praveen, C. S.; Borosy, A. P.; Coperet, C.; Comas-Vives, A., Strain in Silica-Supported Ga(III) Sites: Neither Too Much nor Too 
Little for Propane Dehydrogenation Catalytic Activity. Inorg Chem 2021.

22. Sohn, H.; Camacho-Bunquin, J.; Langeslay, R. R.; Ignaciode Leon, P. A.; Niklas, J.; Poluektov, O. G.; Liu, C.; Connell, J. G.; Yang, D.; Kropf, J.; Kim, H.; Stair, P. C.; Ferrandon, M.; Delferro, M., Isolated, well-defined organovanadium(iii) on silica: single-site catalyst for hydrogenation of alkenes and alkynes. Chemical Communications 2017, 53 (53), 7325-7328.

23. Kaphan, D. M.; Ferrandon, M. S.; Langeslay, R. R.; Celik, G.; Wegener, E. C.; Liu, C.; Niklas, J.; Poluektov, O. G.; Delferro, M., Mechanistic Aspects of a Surface Organovanadium(III) Catalyst for Hydrocarbon Hydrogenation and Dehydrogenation. ACS Catal 2019, 9 (12), 11055-11066.

24. Islam, M. M.; Costa, D.; Calatayud, M.; Tielens, F., Characterization of Supported Vanadium Oxide Species on Silica: A Periodic DFT Investigation. The Journal of Physical Chemistry C 2009, 113 (24), 10740-10746.

25. Liu, C.; Camacho-Bunquin, J.; Ferrandon, M.; Savara, A.; Sohn, H.; Yang, D.; Kaphan, D. M.; Langeslay, R. R.; Ignacio-de Leon, P. A.; Liu, S.; Das, U.; Yang, B.; Hock, A. S.; Stair, P. C.; Curtiss, L. A.; Delferro, M., Development of activity-descriptor relationships for supported metal ion hydrogenation catalysts on silica. Polyhedron 2018, 152, 73-83.

26. Feher, F. J.; Newman, D. A.; Walzer, J. F., Silsesquioxanes as models for silica surfaces. Journal of the American Chemical Society 1989, 111 (5), 1741-1748.

27. $\quad$ Fraile, J. M.; Garcia, J. I.; Mayoral, J. A.; Vispe, E., Catalytic sites in silica-supported titanium catalysts: silsesquioxane complexes as models. J Catal 2005, 233 (1), 90-99.

28. Quadrelli, E. A.; Basset, J.-M., On silsesquioxanes' accuracy as molecular models for silica-grafted complexes in heterogeneous catalysis. Coordination Chemistry Reviews 2010, 254 (5-6), 707-728.

$29 . \quad$ Das, U.; Zhang, G. H.; Hu, B.; Hock, A. S.; Redfern, P. C.; Miller, J. T.; Curtiss, L. A., Effect of Siloxane Ring Strain and Cation Charge Density on the Formation of Coordinately Unsaturated Metal Sites on Silica: Insights from Density Functional Theory (DFT) Studies. ACS Catal 2015, 5 (12), 7177-7185.

30. Guillo, P.; Lipschutz, M. I.; Fasulo, M. E.; Tilley, T. D., Tantalum-Polyhedral Oligosilsesquioxane Complexes as Structural Models and Functional Catalysts for Epoxidation. ACS Catal 2017, 7 (4), 2303-2312.

31. Plascencia, C.; Curtiss, L. A.; Liu, C., Hydrogen Activation by Silica-Supported Metal Ion Catalysts: Catalytic Properties of Metals and Performance of DFT Functionals. Journal of Physical Chemistry A 2019, 123 (1), 171-186.

32. Frisch, M. J.; Trucks, G. W.; Schlegel, H. B.; Scuseria, G. E.; Robb, M. A.; Cheeseman, J. R.; Scalmani, G.; Barone, V.; Petersson, G. A.; Nakatsuji, H.; Li, X.; Caricato, M.; Marenich, A. V.; Bloino, J.; Janesko, B. G.; Gomperts, R.; Mennucci, B.; Hratchian, H. P.; Ortiz, J. V.; Izmaylov, A. F.; Sonnenberg, J. L.; Williams; Ding, F.; Lipparini, F.; Egidi, F.; Goings, J.; Peng, B.; Petrone, A.; Henderson, T.; Ranasinghe, D.; Zakrzewski, V. G.; Gao, J.; Rega, N.; Zheng, G.; Liang, W.; Hada, M.; Ehara, M.; Toyota, K.; Fukuda, R.; Hasegawa, J.; Ishida, M.; Nakajima, T.; Honda, Y.; Kitao, O.; Nakai, H.; Vreven, T.; Throssell, K.; Montgomery Jr., J. A.; Peralta, J. E.; Ogliaro, F.; Bearpark, M. J.; Heyd, J. J.; Brothers, E. N.; Kudin, K. N.; Staroverov, V. N.; Keith, T. A.; Kobayashi, R.; Normand, J.; Raghavachari, K.; Rendell, A. P.; Burant, J. C.; Iyengar, S. S.; Tomasi, J.; Cossi, M.; Millam, J. M.; Klene, M.; Adamo, C.; Cammi, R.; Ochterski, J. W.; Martin, R. L.; Morokuma, K.; Farkas, 0.; Foresman, J. B.; Fox, D. J. Gaussian 16 Rev. C.01, Wallingford, CT, 2016.
33. Becke, A. D., Density-functional thermochemistry. III The role of exact exchange. Journal of Chemical Physics 1993, 98 5648-5652.

34. Lee, C.; Yang, W.; Parr, R. G., Development of the ColleSalvetti correlation-energy formula into a functional of the electron density. Physical Review B: Condensed Matter 1988, 37 (2), 785789.

35. Stevens, W. J.; Basch, H.; Krauss, M., Compact effective potentials and efficient shared-exponent basis sets for the first- and second-row atoms. Journal of Chemical Physics 1984, 81 (12), 6026-6033.

36. Stevens, W. J.; Krauss, M.; Basch, H.; Jasien, P. G., Relativistic compact effective potentials and efficient, shared-exponent basis sets for the third-, fourth-, and fifth-row atoms. Canadian Journal of Chemistry 1992, 70 (2), 612-630.

37. Cundari, T. R.; Stevens, W. J., Effective core potential methods for the lanthanides. Journal of Chemical Physics 1993, 98, $5555-5565$.

38. Schäfer, A.; Huber, C.; Ahlrichs, R., Fully optimized contracted Gaussian basis sets of triple zeta valence quality for atoms Li to Kr. Journal of Chemical Physics 1994, 100 (8), 5829-5835.

39. Serebrinsky, S. A., Physical time scale in kinetic Monte Carlo simulations of continuous-time Markov chains. Phys Rev E Stat Nonlin Soft Matter Phys 2011, 83 (3 Pt 2), 037701.

40. Andersen, M.; Panosetti, C.; Reuter, K., A Practical Guide to Surface Kinetic Monte Carlo Simulations. Front Chem 2019, 7, 202

41. Prats, H.; Posada-Pérez, S.; Rodriguez, J. A.; Sayós, R.; Illas, F., Kinetic Monte Carlo Simulations Unveil Synergic Effects at Work on Bifunctional Catalysts. ACS Catal 2019, 9 (10), $9117-$ 9126.

42. Lu, H. P.; Xun, L.; Xie, X. S., Single-molecule enzymatic dynamics. Science 1998, 282 (5395), 1877-82.

43. Xu, W.; Kong, J. S.; Yeh, Y. T.; Chen, P., Single-molecule nanocatalysis reveals heterogeneous reaction pathways and catalytic dynamics. Nat Mater 2008, 7 (12), 992-6.

44. Ye, R.; Mao, X.; Sun, X.; Chen, P., Analogy between Enzyme and Nanoparticle Catalysis: A Single-Molecule Perspective. ACS Catal 2019, 9 (3), 1985-1992.

45. Bai, S.; Zhou, D.; Davis, M. J.; Skodje, R. T., Sum over Histories Representation for Chemical Kinetics. J Phys Chem Lett 2015, 6 (1), 183-8.

46. Wells, R. H.; Gu, X.-K.; Li, W.-X.; Skodje, R. T., Understanding Surface Catalyzed Decomposition Reactions Using a Chemical Pathway Analysis. The Journal of Physical Chemistry C 2018, 122 (49), 28158-28172.

47. Wells, R. H., et. al. (In preparation).

48. Rataboul, F.; Baudouin, A.; Thieuleux, C.; Veyre, L.; Coperet, C.; Thivolle-Cazat, J.; Basset, J. M.; Lesage, A.; Emsley, L., Molecular understanding of the formation of surface zirconium hydrides upon thermal treatment under hydrogen of $\left[(\equiv \mathrm{SiO}) \mathrm{Zr}\left(\mathrm{CH}_{2}{ }^{\mathrm{t}} \mathrm{Bu}\right)_{3}\right]$ by using advanced solid-state NMR techniques. J Am Chem Soc 2004, 126 (39), 12541-50.

49. Curtin, D. Y., Stereochemical control of organic reactions differences in behaviour of diastereoisomers. Rec. Chem. Prog 1954, 15, 110-128.

50. Hammett, L. P., Physical Organic Chemistry. McGrawHill: New York, 1970.

51. Michaelis, L.; Menten, M. L., Die kinetik der invertinwirkung. Biochem. z 1913, 49 (333-369), 352. 

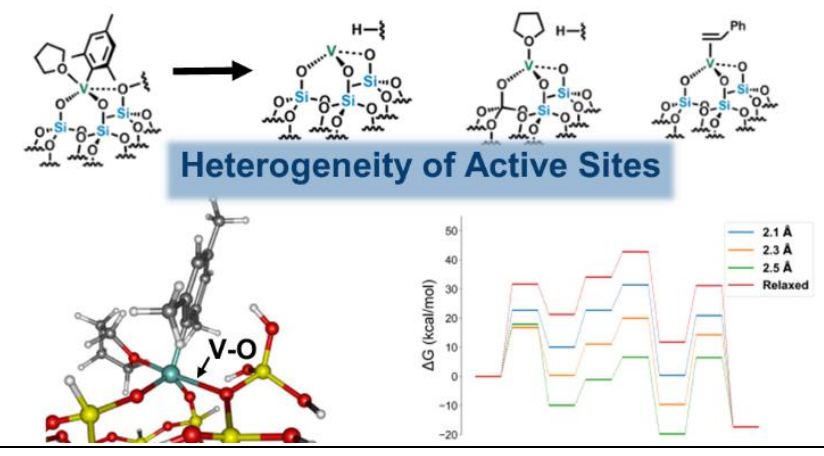